\title{
Quantum Correlated Interstitials and the Hall Resistivity of the Magnetically Induced Wigner Crystal
}

\author{
Lian Zheng and H.A. Fertig \\ Department of Physics and Astronomy, University of Kentucky, Lexington, Kentucky 40506-0055
}

(April 20, 2022)

\begin{abstract}
We study a trial wavefunction for an interstitial in a Wigner crystal. We find that the electron correlations, ignored in a conventional Hartree-Fock treatment, dramatically lower the interstitial energy, especially at fillings close to an incompressible liquid state. The correlation between the interstitial electron and the lattice electrons at $\nu<1 / m$ is introduced by constructing a trial wavefunction which bears a Jastrow factor of a Laughlin state at $\nu=$ $1 / m$. For fillings close to but just below $\nu=1 / m$, we find that a perfect Wigner crystal becomes unstable against formation of such interstitials. It is argued that conduction due to correlated interstitials in the presence of weak disorder leads to the classical Hall resistivity, as seen experimentally.

73.40.Hm, 73.20.Dx
\end{abstract}

Typeset using REVTEX 
In very strong perpendicular magnetic fields at low temperatures, it is generally believed that a two-dimensional electron gas should form a crystalline lattice, in the absence of disorder. A growing body of experimental evidence has accumulated in the last five years that this so-called "Wigner crystal" [1] (WC) may have been realized in high-quality heterojunction systems. Evidence of this has come in the form of rf data [2, [3], transport experiments [4], cyclotron resonance [0], and photoluminescence (PL) experiments [6,7]. One of the very interesting aspects of this system is the competition between the quantum mechanical correlations that lead to the fractional quantum Hall effect (FQHE), which are elegantly described by the Laughlin trial wavefunctions [8], and the types of wavefunctions that describe the Wigner crystal, which is typically described by a Hartree or Hartree-Fock (HF) approximation as a starting point [9]. In particular, transport data [4 have suggested that there is a very sharp transition between the FQHE and the WC as one varies the magnetic field in the vicinity of filling fraction $\nu=1 / 5$. Since the transition occurs over an extremely narrow magnetic range, it is natural to ask whether there are any fluctuations in the crystal state that hint at the transition if one is at filling fractions (for example) near, but below the transition. We address this subject in this Letter.

Our principle motivation for this study is the expectation that density fluctuations of the WC in the vicinity of a FQHE filling fraction should be strongly influenced by Laughlin-like correlations. As a paradigm of this, consider the WC at filling fractions close to, but just below $\nu=1 / 5$. Among the basic excitations of the WC, interstitial electrons in particular create a local charge density that is raised closer to, or possibly above, $1 / 5$. We expect that interstitial energies should be strongly renormalized by correlations in this situation [10]. To model such effects, we consider the following trial wavefunction for a WC with a single interstitial:

$$
\psi=\mathcal{A}\left\{\prod_{i=1}^{N}\left(u-z_{i}\right)^{m} \mathrm{e}^{-|u|^{2} / 4} \psi_{W C}\left(z_{1}, \ldots, z_{N}\right)\right\}
$$

where $\psi_{W C}\left(z_{1}, \ldots, z_{N}\right)$ is the wavefunction for a perfect WC of $N$ electrons, with electrons localized near sites $\vec{R}_{i}^{o}, z_{i}=x_{i}-i y_{i}$ is an electron coordinate in complex notation, $u$ 
is the interstitial coordinate, $m$ is an integer chosen such that $1 / m$ is larger than the filling fraction $\nu$ (we show below that the wavefunction no longer represents an interstitial for $\nu>1 / m)$, and $\mathcal{A}$ is the antisymmetrization operator. We work here in units of the magnetic length $l_{o}=(\hbar c / e B)^{1 / 2}$, where $B$ is the applied magnetic field. We note that if one uses a Hartree approximation for $\psi_{W C}$, the case $m=0$ corresponds to an antisymmetrized Hartree approximation for the interstitial. Such approximate forms for wavefunctions of the $\mathrm{WC}$ at low enough fillings have been argued to be quite good, since one may show that exchange corrections to the WC energy at low fillings are quite small [9].

We have evaluated the energy of the wavefunction in Eq. (1) for a Coulomb potential approximately for various fillings and choices of $m$. Fig. [illustrates these energies for $m=5$ and $m=7$ as a function of $\nu$. The energy $\epsilon$ plotted here is defined as $\epsilon=E_{N+1}^{i}-E_{N+1}^{W C}$, where $E_{N+1}^{i}$ is the energy of the state with $N$ electrons and 1 interstitial, $E_{N+1}^{W C}$ is the energy of a perfect lattice of $N+1$ electrons. Thus, $\epsilon$ represents the energy to deform a perfect lattice of $N+1$ electrons into a lattice with $N$ electrons and one interstitial, with no change in the net charge of the system. For simplicity, we have used a square rather than a triangular lattice for $\psi_{W C}$; this should have no effect on our basic conclusions. In the insert of Fig. [1, the energy $\epsilon$ is shown as a function of the strength of the correlation parameter $m$. One can see in the main figure that the energies are extremely low over a large range of filling fractions, and clearly become negative for filling fractions just below $\nu=1 / m$. (For the case $m=5$, the energy is just slightly negative over a surprisingly large range of $\nu$. Our numerical error unfortunately does not allow us to conclude that the energies are actually negative except above $\nu \approx 0.17$ ). We have found similar results for $\nu=1 / 9$.

Several important conclusions immediately follow from these results: (1) A perfect Hartree-Fock WC cannot be stable for filling fractions very close to $\nu=1 / m$ for $m \leq 9$. Our calculations indicate that, at the very least, a superlattice state of correlated interstitials would have lower energy than a perfect HF WC at such fillings. We will argue below that simply including a Jastrow factor in front of an otherwise perfect HF WC will not lead to such a large lowering of the energy. Such a structural phase transition may be related to PL 
anomalies seen for $\nu=1 / m$, but at much lower fillings than those for which the FQHE is found in transport [11]. (2) Since the energies of the interstitial states are so very low over a large range of filling fractions, these are likely to contribute significantly to charge transport at fillings for which the crystal is stable. Furthermore, we will argue below that the Hall resistivity in the presence of weak disorder takes on the classical value $\rho_{x y}=h / \nu e^{2}$, as is found in experiment. 17] (3) Experimental observation of a vanishing activation energy as the filling fraction approaches $1 / 5$ from below could be understood in terms of the correlated interstitials, whose energies vanish near the FQHE filling fractions. (4) The well-known [12] "dip" in $\rho_{x x}$ near $\nu=1 / 7$, where to date no FQHE has been observed, might be understood as being due to correlated interstitials, which have particularly low energies near this filling.

To understand why the correlated interstitial has such low energy, it is convenient to use a variation of the "plasma analogy" first exploited by Laughlin in the context of the FQHE [8]. Dropping the antisymmetrization [13] in Eq. (11), and using a Hartree approximation for $\psi_{W C}$, we write the square modulus in the form $|\psi|^{2}=e^{-\beta \Phi}$, where, for $\beta=1$,

$$
\Phi=-2 m \sum_{i=1}^{N} \ln \left(\left|u-z_{i}\right|\right)+\frac{|u|^{2}}{2}+\sum_{i=1}^{N} \frac{\left|z_{i}-R_{i}^{o}\right|^{2}}{2}
$$

where $R_{i}^{o}=R_{i x}^{o}-i R_{i y}^{o}$ are the perfect WC lattice sites in complex notation. This represents the energy of a classical two-dimensional Coulomb particle with charge 1, interacting with a neutralizing background of charge density $\sigma_{b}=1 / 2 \pi$, and a lattice of charges $m$ with charge density $\sigma_{l}=m / a^{2}$, where $a$ is the WC lattice constant. On a coarse scale, the interstitial is thus interacting with a system of net charge density $\sigma=(1-m \nu) / 2 \pi(\nu$ is the filling in the absence of the interstitial), and thus spreads out in the center of the disk to an area of $1 / \sigma$. We note that precisely at the filling $\nu=1 / m$, the interstitial will "spread out" uniformly over the disk, and for higher fillings the interstitial electron will have its highest probability at the system edge, essentially being ejected from the system. This is the reason the wavefunctions are only physically reasonable for fillings below $\nu=1 / m$ [16]. Fig. 2 illustrates the probability of finding the interstitial at a particular position along a crystal symmetry axis for $m=5$ and various values of $\nu$, which is calculated using Eq. (3) below. 
We are now in a position to understand why the correlations lower the energy of interstitial, especially close to $\nu=1 / m$. In essence, the Jastrow factor allows us to take advantage of quantum fluctuations in the WC. When there are configurations in $\psi_{W C}^{N}$ that have large holes in them, the interstitial has a high probability of being located there, especially when $\nu \sim 1 / m$, for which the interstitial "density" (Fig. 2) samples a large region of the crystal. Conversely, when the crystal forms regions of high density, the interstitial has a low probability of approaching that region. In this way, the interstitial electron does an excellent job of minimizing its energy. We note that a Jastrow factor of exponent $m$, involving all electron pairs, multiplying a perfect WC HF state would probably not lower the energy per electron so much as the interstitial electron energy is lowered when $\nu<1 / m$. This is because in the former case the electrons tend to remain closely tied to their lattice sites, and hence do not "sample" enough of the lattice to take great advantage of the quantum fluctuations. Thus, the low energy of the interstitial wavefunction is due as much to its locally high density as it is to the good correlations it has with the lattice electrons.

We now consider the Hall effect due to correlated interstitials in the presence of weak disorder, and show this gives the classical Hall resistivity, as seen in experiment. [17] We first need to determine how disorder is likely to affect our interstitial wavefunction. The main source of disorder in heterostructures is thought to be caused by fluctuations in the neutralizing background charge density, which typically is set back from the electron gas by several thousand Angstroms. Recent classical calculations of the electron crystal state in the presence of such disorder have shown that this leads to small variations of the crystal density over long length scales. [18] As seen in Fig. 1, the interstitial energetically favors regions of higher density. We can build correlations into the wavefunctions to optimize the energy by altering our correlation factor slightly, to take the form $\prod_{i}\left(u-z_{i}\right)^{m_{i}}$, with $m_{i}$ chosen to be larger for lattice sites in low density regions, and smaller elsewhere. One may show that the interstitial will not be ejected from the system, provided $<m_{i}>\leq 1 / \nu$, where $<\ldots>$ denotes an average over lattice sites. The higher energy regions may be optimally avoided if one chooses $\left.<m_{i}\right\rangle=1 / \nu$. 
The Hall resistivity of interstitials in such states can be estimated using a mean-field approximation, in which we replace $m_{i}$ by $\left\langle m_{i}\right\rangle$ for all the lattice sites. This is justified, because if the interstitials are flowing in a current, they will pass many of the lattice electrons. Furthermore, the correlation factors turn out to affect the lattice electrons over very long distances, as will be described below. The key insight is that the correlation factor $\left(u-z_{i}\right)^{m_{i}}$ makes the interstitial appear as a magnetic flux tube to lattice electron $i$, with strength $m_{i} \phi_{0}$, where $\phi_{0}=h c / e$. Thus, on average, a single correlated interstitial flowing through the system should have the same effect as a moving flux tube of strength $\left\langle m_{i}\right\rangle=1 / \nu$. It is easy to show using Faraday's law that an electric current $I$ flowing in the $\hat{x}$ direction in which each carrier has this magnetic flux will generate a force on the lattice electrons, which may be described by an effective voltage drop of magnitude $\frac{h}{\nu e^{2}} I$. This results in a finite Hall resistivity $\rho_{x y}=\frac{h}{\nu e^{2}}$, as observed experimentally. [17] We note that a similar mechanism leads to the finite Hall resistance of the recently proposed "Hall insulator". 19]

We now discuss in some detail how the energy of the interstitial state is evaluated. Using Hartree approximation for $\psi_{W C}$ in Eq. (四) and neglecting the anti-symmetrization, one has

$$
\left|\psi\left(u, z_{1}, \ldots, z_{N}\right)\right|^{2}=\mathrm{e}^{-|u|^{2} / 2} \prod_{i=1}^{N}\left|u-z_{i}\right|^{2 m} \mathrm{e}^{-\left|z_{i}-R_{i}^{o}\right|^{2} / 2}
$$

where $R_{i}^{o}$ forms a square lattice, which would be the center of mass of each lattice electron if the Jastrow-type correlation factor in the wavefunction were absent. The interstitial electron density distribution $\rho(u)=\int \prod_{i=1}^{N} d^{2} z_{i}\left|\psi\left(u, z_{1}, \ldots, z_{N}\right)\right|^{2} / \int d^{2} u \prod_{i=1}^{N} d^{2} z_{i}\left|\psi\left(u, z_{1}, \ldots, z_{N}\right)\right|^{2}$, shown in Fig. 2, can be directly evaluated from the above expression, since the integrations over the lattice electron coordinates may be computed analytically. The energy of this interstitial state $E_{N+1}^{i}$ consists of two parts: $E_{N+1}^{i}=E_{1}+E_{2}$, where $E_{1}$ represents the Coulomb interaction between the interstitial electron and the lattice electrons, and $E_{2}$ represents the pairwise interactions among the lattice electrons. Numerical evaluation of $E_{1}$ can be carried out directly from Eq. (3) [14 by analytical integration over the $z_{i}$ 's and numerical integration over $u$. An exact calculation for $E_{2}$, on the other hand, is highly impractical. The reasons are easily recognized. To obtain the interaction energy from one pair of electrons, 
one needs to compute a 6-dimensional integral over the coordinates of the interstitial and the pair of the electrons involved. This 6-dimensional integral has to be performed for each of the $(1 / 2) N(N-1)$ pairs for a system of $N$ electrons. Moreover, one needs to compute $E_{2}$ for relatively large systems, since $E_{2}$ turns out to depend on the system size [15]. We thus turn to an approximate evaluation of $E_{2}$.

Our approximation scheme is based on the observation that the main effect of the Jastrow factor in Eq. (3) is to shift the center of mass of each lattice electron wavepacket away from $R_{i}^{o}$. Beyond this, these wavepackets are also deformed from circularly symmetric Gaussian distributions. Such effects can be systematically accounted for if we express the Coulumb potential in terms of a multipole expansion. One immediately realizes that the effect of the center of mass shift on $E_{2}$ is much more important than that of the wavepacket deformation. This is because the shift of the center of mass of each lattice electron changes the dipole and higher-order multipole moments, while the wavepacket deformation changes only the quadrupole and higher- order multipole moments. Since the extension of the electron wavepacket is much smaller than the inter-particle distance for $\nu \leq 1 / 5$, a natural approximation for $E_{2}$ is to neglect the wavepacket deformation, ie., to assume that an electron at lattice site $i$ consists of a Gaussian wavepacket localized at the shifted center of mass $\left\langle\vec{r}_{i}\right\rangle$, where $\left\langle\vec{r}_{i}\right\rangle$ is calculated numerically from the probability distribution Eq. (3) as $<\vec{r}_{j}>=\int d^{2} u \prod_{i} d^{2} z_{i} \vec{r}_{j}|\psi|^{2} / \int d^{2} u \prod_{i} d^{2} z_{i}|\psi|^{2}$. Therefore, one has $E_{2} \simeq \sum_{i<j} V\left(\left|<\vec{r}_{i}>-<\vec{r}_{j}>\right|\right)$, where the effective potential is taken as that of a HF crystal 9

$$
V(R)=\frac{1}{R}+\frac{1}{R^{3}}+\frac{9}{2 R^{5}}+\frac{75}{2 R^{7}}+\ldots
$$

The energy of the correlated interstitial state with $E_{1}$ calculated from Eq. (3) and $E_{2}$ calculated from Eq. (4) is shown in Fig. 11. This is our primary result. Before concluding the description for the calculation, we remind the reader that the numerical uncertainties, namely, neglecting the anti-symmetrization, neglecting part of the higher-order multipole contribution to $E_{2}$, as well as the numerical errors, are all relatively small. More important 
corrections to the precise energies of the interstitials would result from using a triangular lattice, and accounting for lattice relaxation in the vicinity of the interstitial. However, such improvements should not alter the qualitative result.

To summarize, we have studied a trial wavefunction for an interstitial in a Wigner crystal and showed that the electron correlations, ignored in a conventional Hartree-Fock treatment, may be essential in understanding several properties of a Wigner crystal, especially at fillings close to an incompressible liquid state. As a result of the correlation, the state of a Wigner crystal with interstitials is found to have very low energy compared to the corresponding Hartree-Fock interstitial. In particular, we have found that for fillings close to but below $\nu=1 / m$, the correlated interstitial state is actually energetically favored over a perfect Wigner crystal. Away from such fillings, we found in the presence of weak disorder that thermally excited interstitials carrying a current generate a transverse electric field, leading to a finite Hall resistivity, $\rho_{x y}=h / \nu e^{2}$, as observed experimentally.

The authors would like to thank Dr. A.H. MacDonald for helpful discussion. This work is supported by NSF through Grant No. DMR-9202255. 


\section{REFERENCES}

[1] E.P. Wigner, Phys. Rev. 46, 1002 (1934)

[2] E.Y. Andrei, G. Deville, D.C. Glattli, F.I.B. Williams, E. Paris, and B. Etienne, Phys. Rev. Lett. 60, 2765 (1988); F.I.B. Williams et al., Phys. Rev. Lett. 66, 3285 (1991)

[3] M.A. Paalanen, R.L. Willett, P.B. Littlewood, K.W. West, L.N. Pfeiffer, and D.J. Bishop, Phys. Rev. B 45, 11342 (1992)

[4] V.J. Goldman, M. Santos, M. Shayegan, and J.E. Cunningham, Phys. Rev. Lett. 65, 2189 (1990); H.W. Jiang, R.L. Willett, H.L. Stormer, D.C. Tsui, L.N. Pfeiffer, and K.W. West, Phys. Rev. Lett. 65, 633 (1990); Y.P. Li, T. Sajoto, L.W. Engel, D.C. Tsui, and M. Shayegan, Phys. Rev. Lett. 67, 1630 (1991)

[5] M. Besson, E. Gornick, C.M. Engelhardt, and G. Weimann, Semiconductor Sci. Technol. $7,1274(1992)$

[6] H. Buhmann et al., Phys. Rev. Lett. 66, 926 (1991); Phys. Rev. B 45, 4532 (1992)

[7] E.M. Goldys et al., Phys. Rev. B 46, 7957 (1992); R.G. Clark, Physica Scripta T39, 45 (1991)

[8] R.B. Laughlin, Surf. Sci. 142, 163 (1984)

[9] See, for example, K. Maki and X. Zotos, Phys. Rev. B 28, 4349 (1983); R. Côté and A.H. MacDonald, Phys. Rev. Lett. 69, 816 (1992); P.K. Lam and S.M. Girvin, Phys. Rev. B 30, 473 (1984); D. Levesque, J.J. Weis, and A.H. MacDonald, Phys. Rev. B 30, 1056 (1984); Xuejun Zhu and Steven G. Louie, Phys. Rev. Lett. 70, 335 (1993)

[10] Among the basic excitations of the WC, the interstitial has points of higher local density than other types of excitations. We thus expect it to be more strongly affected by correlations than other excitations below but near a FQHE filling fraction.

[11] H. Buhmann et al., Phys. Rev. Lett. 65, 1056 (1990) 
[12] V.J. Goldman, M. Shayegan, and D.C. Tsui, Phys. Rev. Lett. 61, 881 (1988)

[13] We have estimated the exchange correction to the energy. Due to the Jastrow factor, the correction to the energy from wavefunction overlap between the interstitial and any of its nearest lattice electrons is in the order of 1 part in $10^{6}$ for $\nu<1 / 5$. The small effects of exchange between two lattice electrons have been known from the work of Maki and Xotos, Ref. [9].

[14] For a system size of $N \geq 4 \times 10^{4}$, the accuracy of $E_{1}$ is better than $0.01 \mathrm{e}^{2} / l_{o}$.

[15] The system size has to be large enough to eliminate the boundary effects on $E_{2}$. In the actual calculation of $E_{2}$ described in the following paragraphs, the system size is chosen as $N=(1 / 5,2 / 5,3 / 5,4 / 5,1) \times 10^{6}$. The value of $E_{2}$ is then extrapolated to $N=\infty$.

[16] It is interesting to speculate that similar states could be constructed for vacancies for fillings just above $\nu=1 / m$.

[17] V.J. Goldman, J.K. Wang, Bo Su, and M. Shayegan, Phys. Rev. Lett. 70, 647 (1993)

[18] M.C. Cha and H.A. Fertig, unpublished.

[19] S. Kivelson, D.H. Lee, and S.C. Zhang, Phys. Rev. B 462223 (1992) 


\section{FIGURES}

FIG. 1.

The energy $\epsilon=E_{N+1}^{i}-E_{N+1}^{W C}$ as functions of the Landau level filling factor $\nu$ for $m=5$ and $m=7$, where $E_{N+1}^{i}$ is the energy of the $N+1$ electron state described by Eq. (3) and $E_{N+1}^{W C}$ is the energy of a $N+1$ electron Hartree-Fock Wigner crystal state. The insert shows the energy $\epsilon$ at $\nu=0.19$ as a function of the exponent $m$ of the Jastrow factor, ie., as a function of the strength of the correlation.

FIG. 2 .

The probability distribution for the interstitial electron in a correlated interstitial state described by Eq. (33) with $m=5$ and $\nu=0.19,0.17,0.1$. The distance is along the $y=0$ axis in a Cartesian coordinate reference frame where the square lattice $R_{i j}^{o}$ is expressed as $(i-0.5, j-0.5)$. The distance is in unit of the lattice constant $a$. 